\title{
Pig genome sequence - analysis and publication strategy
}

\author{
Alan L Archibald ${ }^{1 *}$, Lars Bolund ${ }^{2,3}$, Carol Churcher ${ }^{4}$, Merete Fredholm5, Martien AM Groenen ${ }^{6}$, Barbara Harlizius ${ }^{7}$, \\ Kyung-Tai Lee ${ }^{8}$, Denis Milan ${ }^{9}$, Jane Rogers ${ }^{10}$, Max F Rothschild ${ }^{11}$, Hirohide Uenishi ${ }^{12}$, Jun Wang ${ }^{2,13}$, \\ Lawrence B Schook $^{14^{*}}$, the Swine Genome Sequencing Consortium
}

\begin{abstract}
Background: The pig genome is being sequenced and characterised under the auspices of the Swine Genome Sequencing Consortium. The sequencing strategy followed a hybrid approach combining hierarchical shotgun sequencing of BAC clones and whole genome shotgun sequencing.

Results: Assemblies of the BAC clone derived genome sequence have been annotated using the Pre-Ensembl and Ensembl automated pipelines and made accessible through the Pre-Ensembl/Ensembl browsers. The current annotated genome assembly (Sscrofa9) was released with Ensembl 56 in September 2009. A revised assembly (Sscrofa10) is under construction and will incorporate whole genome shotgun sequence (WGS) data providing > 30x genome coverage. The WGS sequence, most of which comprise short Illumina/Solexa reads, were generated from DNA from the same single Duroc sow as the source of the BAC library from which clones were preferentially selected for sequencing. In accordance with the Bermuda and Fort Lauderdale agreements and the more recent Toronto Statement the data have been released into public sequence repositories (Genbank/EMBL, NCBI/Ensembl trace repositories) in a timely manner and in advance of publication.
\end{abstract}

Conclusions: In this marker paper, the Swine Genome Sequencing Consortium (SGSC) sets outs its plans for analysis of the pig genome sequence, for the application and publication of the results.

\section{Background}

The pig genome is being sequenced and characterised under the auspices of the Swine Genome Sequencing Consortium [1]. A Data Release Workshop convened in Toronto in May 2009 by Genome Canada and other funding agencies affirmed and extended the commitments to prepublication release of large data sets in the life sciences which were originally developed in the context of the Human Genome Project. The Toronto Statement [2] places obligations on the producers of such data sets, including genome sequence data, in respect of prepublication release of the data and confirms the principle that allows the data producers to publish the first global analyses of the data set. The data producers are encouraged to produce a citable statement or "marker paper" in which they describe the data set and their

\footnotetext{
* Correspondence: alan.archibald@roslin.ed.ac.uk; schook@illinois.edu

${ }^{1}$ The Roslin Institute and Royal (Dick) School of Veterinary Studies, University of Edinburgh, Roslin, UK

${ }^{14}$ Institute of Genomic Biology, University of Illinois, Urbana, Illinois, USA
}

intentions in respect of analysis and publication. In this marker paper, the Swine Genome Sequencing Consortium (SGSC) sets outs its plans for analysis of the pig genome sequence, for the application and publication of the results. These plans were presented to participants in the Pig Genome III conference held at the Wellcome Trust Sanger Institute, 2-4 November 2009.

\section{Results}

\section{Pig genome sequence data}

The sequence data from which a draft pig genome sequence will be assembled comprises hierarchical shotgun sequence data providing 4-6x genome coverage from BAC clones representing a minimal tile path across the genome plus $>30 \times$ genome coverage in whole genome shotgun sequence (WGS) data generated using Sanger (capillary) and next-gen (Illumina) technologies. The minimal tile path was identified from a high quality physical (BAC contig) map [3] and provides coverage of $98.3 \%$ of this physical map. As at $5^{\text {th }}$ July 2010

\section{Biomed Central}


the total length of the BAC-derived sequence contigs, prior to the removal of sequence redundancy between overlapping BAC clones, was $3.01 \mathrm{Gbp}$ of which 156.3 Mbp was at finished quality. These sequence data were generated from 16,707 BAC clones of which 15,895 have been subjected to one round of automated pre-finishing.

\section{Prepublication data release}

In accordance with the Bermuda and Fort Lauderdale agreements and the more recent Toronto Statement [2] the data have been released into public sequence repositories (Genbank/EMBL, NCBI/Ensembl trace repositories) in a timely manner and in advance of publication. Assemblies of the genome sequence have been annotated using the Pre-Ensembl and Ensembl automated pipelines and made accessible through the Pre-Ensembl/Ensembl browsers. The current annotated genome assembly (Sscrofa9) was released with Ensembl 56 in September 2009. The current assembly (Sscrofa9) was constructed entirely from the BAC-derived sequence data.

\section{Analysis strategy}

A revised assembly (Sscrofa10) is being constructed from the BAC clone derived sequence together with the WGS data. The publication of a draft genome sequence for the pig will be based on this new assembly. A series of analysis working groups have been established in consultation with the pig genome research community under the auspices of the SGSC in order to undertake genome-wide analyses of the genome sequence. These groups with their respective lead contacts are summarised in Table 1. Details of the work of these groups will be posted on the SGSC website at http://www.piggenome.org.

\section{Publication strategy}

The Swine Genome Sequencing [1] and Swine HAPMAP [4] consortia respectively propose to develop two summary papers for publication describing a) the sequencing and analysis of the pig genome and $b$ ) genetic variation and haplotype structures across a range of pig breeds and related Sus species. In addition, the consortia propose to develop a series of companion papers describing either the results from the analysis groups and/or results from other research projects that have been enabled by the publication of a draft sequence of the pig genome. The consortia would be pleased to hear from research groups with plans for manuscripts that could be included within the list of companion papers. Please address correspondence to either Alan Archibald alan.archibald@roslin.ed.ac.uk or Larry Schook schook@illinois.edu.

\section{Discussion}

The value of the pig genome sequence lies not only in shaping the continued use of pigs in agriculture and medical research but also in the realm of evolution and domestication (natural and artificial selection) [5]. The pig is an economically important species not only as a major source of meat-based protein but also increasingly as a model for biomedical research. For example, the pig has value as a model of a spectrum of human diseases that may be modelled less well in rodents, including obesity, arthritis and cardiovascular disease.

The domestic pig (Sus scrofa) is a eutherian mammal and a member of the Cetartiodactyla order, a clade distinct from rodent and primates that last shared a common ancestor with humans between 79 and 87 million years ago. The domestic pig belongs to the Suidea family that consists of multiple species, all found in Asia, Europe and Africa. The availability of this wide variety of pig species that diverged over a period of around 2 to 15 million years provides a rich resource to study genomic changes in relation to speciation. A well characterised pig genome sequence forms a template for the study of within and between species genetic variation. Our analysis of the pig genome sequence will be set in the context of parallel research on the genomes of closely related and contemporary Suids (e.g. Sus verrocus, Sus celebensis and Sus barbatus) and on within breed genetic variation using the $60 \mathrm{~K}$ pig SNP chip [4] and by re-sequencing.

\section{Conclusions}

The pig genome sequencing project has been conducted in an open international collaborative manner in the spirit of the Bermuda and Fort Lauderdale agreements. In accordance with the more recent Toronto Statement the sequence data have been released in advance of publication. In this marker paper, the Swine Genome Sequencing Consortium (SGSC) sets outs its plans for analysis of the pig genome sequence, for the application and publication of the results.

\section{Methods}

\section{Sequencing strategy}

The pig genome has been sequenced following a hybrid approach representing a refinement of the strategy announced earlier [1] (Figure 1). Briefly, BAC clones selected to represent a minimal tile path across the genome were identified from the high resolution physical (BAC contig) map [2] and were subjected to hierarchical shotgun sequencing. BAC clones from the CHORI-242 library prepared from DNA from a single Duroc sow (Duroc 2-14) were preferentially chosen for sequencing. The initial plan was to skim sequence the $\mathrm{BAC}$ clones to $3 \times$ coverage. In practice, both ends of 
Table 1 Swine Genome Sequencing Consortium genome sequence analysis groups

\begin{tabular}{lll}
\hline Analysis group & Lead contact & Notes \\
\hline Assembly & Alan Archibald & The target for the next assembly is to incorporate all the available \\
alan.archibald@roslin.ed.ac.uk & $\begin{array}{l}\text { sequence data for Duroc 2-14, including BAC clones sequences, WGS } \\
\text { Sanger and next-generation short sequence reads. Contig and scaffold } \\
\text { order and orientation will be tested against other genome maps and } \\
\text { in particular the high resolution radiation hybrid maps. }\end{array}$
\end{tabular}

Structural variation, segmental duplication, copy number variation

Repetitive DNA, transposable elements Speciation, wild and related suids and selection

Evolution

Comparative genomics

Imprinting

SNP

ncRNA

Gene builds

Protein interactions

Immune genes

Reproduction
Christian Bendixen christian.bendixen@agrsci.dk

Geoff Faulkner geoff.faulkner@roslin.ed.ac.uk Lawrence Schook schook@uiuc.edu

Leif Andersson Leif.andersson@imbim.uu.se

Martien Groenen Martien.groenen@wur.nl

Ole Madsen

Ole.madsen@wur.nl

Martien Groenen martien.groenen@wur.nl

Jan Gorodkin gorodkin@genome.ku.dk

Steve Searle Searle@sanger.ac.uk

Soren Brunak brunak@cbs.dtu.dk

Chris Tuggle cktuggle@iastate.edu

Max Rothschild mfrothsc@iastate.edu
The reference genome sequence will be analysed for evidence of segmental duplications. Comparative Genomic Hybridisation data, paired-end and mate-pair re-sequence data from other pigs will be used to identify smtructural and copy number variation.

Retroviruses and related repetitive sequences in Sus scrofa and related species will be characterized. Sequence and 60 K SNP genotype data from wild boar and related species will be explored to address the origins of domestic pigs. Comparative sequence analyses of domesticated and wild boar genome sequences is expected to reveal signatures of artificial and natural selection.

Natural and artificial selection will have shaped the pig genome sequence. Comparison of the pig genome sequence with the sequences of other mammals is expected to reveal genes that are evolving more rapidly in the pig and artiodactyl lineages.

Genome rearrangements and conserved synteny compared to other suids and other mammals.

RNA-seq data from a range of tissues from Duroc 2-14 or her clones will be analysed to identify genes that show differential allelic expression and potentially imprinted genes.

Re-sequence data and the WGS sequence data from Duroc 2-14 will be examined for putative SNPs and small indels, including those for which Duroc 2-14 is heterozygous.

The genome sequence will be explored for putative ncRNA sequences and microRNA encoding loci.

The Ensembl automated pipeline will be used to establish a Gene Build for the pig genome that will be compared with builds generated by other systems including NCBI.

Development of a proteome will be initiated.

The immune gene analysis group will manually annotate pig genes predicted/known to have roles in the immune system. The repertoire of pig immune genes will be examined for evidence of pig-lineage specific features

The reproduction gene analysis group will manually annotate pig genes predicted/known to have roles in reproductive functions and seek to identify pig-lineage specific features. 


\section{Table 1 Swine Genome Sequencing Consortium genome sequence analysis groups (Continued)}

\begin{tabular}{ll}
\hline Olfaction, neuropeptide & Sandra Rodriguez-Zas \\
and prohormone & rodrgzzs@illinois.edu
\end{tabular}

and prohormone $\quad$ rodrgzzs@illinois.edu

Manual annotation

Biomedical Models
Jim Reecy

jreecy@iastate.edu
Approximately $5 \%$ of the genes in the Sscrofa9 Gene Build are predicted to have olfactory functions. These genes will be manually annotated and examined for pig-specific characteristics. In addition, the neuropeptide and prohormone gene families will be annotated.

The pig research community is engaged in efforts to manually Annotate genes identified/predicted by the Ensembl analysis pipeline. The otterlace system will be used to enable this community annotation activity.

The use of genomic information to enhance the utilization of the pig in xenotransplantation and as a model for cardiovascular, cancer and obesity will be addressed. How genomic information supports the further development of transgenic pigs for creating essential animal models will also be discussed.

768 subclones for each BAC were sequenced (average read length of $707 \mathrm{bp}$ ) to provide $\sim 4 \times$ coverage. Most $\mathrm{BAC}$ clones have subsequently been subjected to one round of automated pre-finishing by primer walking from the ends of the clone sequence contigs constructed from the initial $4 \times$ coverage skim sequencing. This hierarchical shotgun sequencing was primarily undertaken at the Wellcome Trust Sanger Institute, with additional clones sequenced by the National Institute of Agrobiological Sciences, Japan. In addition whole genome shotgun (WGS) sequence data were generated from DNA isolated from the same animal (Duroc 2-14). These WGS data were generated using both Sanger capillary sequencing at the Korean Livestock Research Institute and Illumina/Solexa sequencing at the Beijing Genomics Institute and the Wellcome Trust Sanger Institute.

\section{Acknowledgements}

The Swine Genome Sequencing Consortium is grateful to the following for funding support for the pig genome sequencing project: the USDA National Institute of Food and Agriculture, formerly the Cooperative State Research, Education and Extension Service; the Agence Nationale de la Recherche; European Union SABRE; the Institute for Pig Genetics, Netherlands; INRA Genescope, France; lowa Pork Producers Association; lowa State University; Korean National Livestock Research Institute; National Institute of Agrobiological Sciences, Japan; National Pork Board, U.S.; North Carolina Pork Council; North Carolina Agricultural Research Service; North Carolina State University; the University of Illinois; the "Pigs and Health" programme of the Danish Advanced Technology Foundation, Denmark; the Wellcome Trust Sanger Institute; The Roslin Institute, University of Edinburgh and Biotechnology and Biological Sciences Research Council, U.K.; the University of Illinois Livestock Genome Sequencing Initiative.

\section{Author details}

${ }^{1}$ The Roslin Institute and Royal (Dick) School of Veterinary Studies, University of Edinburgh, Roslin, UK. ${ }^{2}$ BGI-Shenzhen, Shenzhen 518083, China. Institute of Human Genetics, Aarhus University, DK-8000 Aarhus, Denmark. ${ }^{4}$ The Wellcome Trust Sanger Institute, The Wellcome Trust Genome Campus, Hinxton, Cambridge, UK. ${ }^{5}$ Copenhagen University, Copenhagen, Denmark.

${ }^{6}$ Wageningen University, Animal Breeding and Genomics Centre,

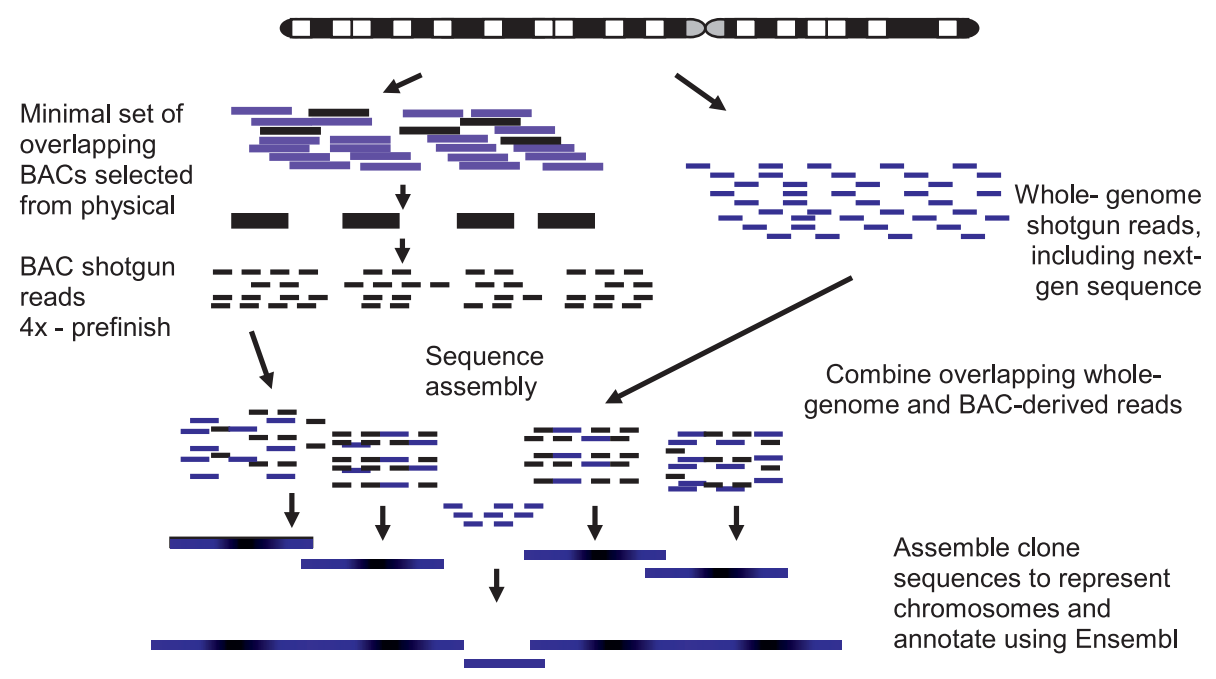

Figure 1 Sequencing strategy - a hybrid approach combining hierarchical shotgun sequencing of BAC clones and whole genome shotgun sequencing. 
Wageningen, The Netherlands. 'Institute for Pig Genetics, Beuningen, The Netherlands. ${ }^{8}$ Korean National Institute of Animal Science, Suwon, Kyunggido, Korea. ${ }^{9}$ INRA Toulouse, France. ${ }^{10}$ The Genome Analysis Centre, Norwich UK. "'Department of Animal Science and Center for Integrated Animal Genomics, lowa State University, Ames, lowa 50011, USA. ${ }^{12}$ National Institute of Agrobiological Sciences, Japan. ${ }^{13}$ Department of Biology, University of Copenhagen, Copenhagen, Denmark. ${ }^{14}$ Institute of Genomic Biology, University of Illinois, Urbana, Illinois, USA.

\section{Authors' contributions}

All authors are members of the Swine Genome Sequencing Consortium (SGSC) under whose auspices the pig genome is being sequenced. They are responsible for securing the funding for, and the management of, the pig genome sequencing project. ALA, DM, JR, MFR and LBS are members of the SGSC Steering Committee. ALA, MF, DM, JR, MFR, HU and LBS are members of the SGSC Technical Committee. LBS, CC, ALA, MAMG, DM, JR, MF, MFR comprise the SGSC Manuscript Steering Committee which is directing the SGSC's analysis and publication strategy. JR and CC led the sequencing team at the Wellcome Trust Sanger Institute which generated the BAC clone derived sequence data, during the initial and later stages of the project, respectively. LBS and JR were co-directors of the USDA grant which provided ca. 50\% of the project funding. MAMG was work package leader for the EC-funded project to sequence chromosomes 7 and 14. BH and MAMG were project leaders for the IPG-funded project to sequence chromosome 4. ALA was the PI for the BBSRC grant on annotation and analysis. MFR secured US pig industry funding for the project and led a pilot project to generate finished sequence for part of chromosome 17. JW led the Beijing Genomics Institute effort to generate WGS data using Illumina next-gen sequencing technology partially funded by a grant of which LB was the PI. K-TL led the team at the Korean Livestock Research Institute that has contributed WGS data using Sanger capillary technology. HU leads the team at Japanese National Institute of Agrobiological Sciences which contributed full length CDNA sequence and some BAC clone sequence data. DM leads the team which is validating the sequence assembly against a high resolution radiation hybrid map. Finally, some of the leadership roles of the authors in the analysis of the sequence data are highlighted in Table 1. All authors have read and approved the manuscript.

Received: 15 April 2010 Accepted: 19 July 2010 Published: 19 July 2010

\section{References}

1. Schook LB, Beever JE, Rogers J, Humphray S, Archibald A, Chardon P, Milan D, Rohrer G, Eversole K: Swine Genome Sequencing Consortium (SGSC): a strategic roadmap for sequencing the pig genome. Comp Funct Genom 2005, 6:251-255.

2. Toronto International Data Release Workshop Authors: Prepublication data release. Nature 2009, 461:168-70

3. Humphray SJ, Scott CE, Clark R, Marron B, Plumb R, Bender C, Camm N, Davis J, Jenks A, Noon A, Patel M, Sehra H, Yang F, Rogatcheva MB, Milan D, Chardon P, Rohrer G, Nonneman D, de Jong P, Meyers SN, Archibald A, Beever JE, Schook $L B$, Rogers J: A high utility integrated map of the pig genome. Genome Biol 2007, 8(7):R139.

4. Ramos AM, Crooijmans RPMA, Amaral AJ, Archibald AL, Beever JE, Bendixen C, Dehais P, Affara NA, Hansen MS, Hedegaard J, Hu Z-L, Kerstens HH, Law AS, Megens HJ, Milan D, Nonneman DJ, Rohrer GA, Rothschild MF, Smith TPL, Schnabel RD, Van Tassell CP, Clark R, Churcher C, Taylor JF, Wiedmann RT, Schook LB, Groenen MAM: Design of a high density SNP genotyping assay in the pig using SNPs identified and characterized by next generation sequencing technology. PLOS ONE 4(8): e6524.

5. Rohrer G, Beever JE, Rothschild MF, Schook L, Gibbs R, Weinstock G: Porcine genomic sequencing initiative. (NIH White Paper) 2002 [http:// www.animalgenome.org/pigs/community/PigWhitePaper/].

doi:10.1186/1471-2164-11-438

Cite this article as: Archibald et al:: Pig genome sequence - analysis and publication strategy. BMC Genomics 2010 11:438.

\section{Submit your next manuscript to BioMed Central and take full advantage of:}

- Convenient online submission

- Thorough peer review

- No space constraints or color figure charges

- Immediate publication on acceptance

- Inclusion in PubMed, CAS, Scopus and Google Scholar

- Research which is freely available for redistribution

Submit your manuscript at www.biomedcentral.com/submit
Ciomed Central 